\title{
Lecture on aberrations
}

This content has been downloaded from IOPscience. Please scroll down to see the full text. 1899 Trans. Opt. Soc. 116

(http://iopscience.iop.org/1475-4878/1/1/303)

View the table of contents for this issue, or go to the journal homepage for more

Download details:

IP Address: 130.126.162.126

This content was downloaded on 02/10/2015 at $11: 25$

Please note that terms and conditions apply. 


\title{
LECTURE ON ABERRATIONS
}

\author{
By Professor Stlvanus P. Thompson, B.A., D.Sc., F.R.S., \\ Tice-President.
}

At a Meeting of the Optical Society on January 18th, 1900.

MR. PRESIDENT and gentlemen : When you did me the honour of asking me to give a lecture to the Optical Society, I
happened to be in the position of having just given a half dozen lectures in this theatre to my Friday evening class, and it occurred to me that some one of the topics that I have taken in these lectures might be a suitable one for the Optical Society. I took advice on the subject and I was told that the lecture on Aberrations would probably be the one which appealed most to you, and therefore I propose to talk to you about aberrations.

My object is not to lay down the law for you, but rather to illustrate by experiment in such a manner as one can when one has a lecture theatre fitted up with special appliances, -and apparatus which has been used again and again for optical demonstrations-illustrating by experiment things which very often are not known from that source, but are only learned from books. Those who, whether they are connected with the optical trades or not, are obliged to read up the parts of optics that they do not naturally come across in everyclay work, do not always have the opportunity to grasp fully that which the books describe, because they have never possibly seen the experiments shown. I think therefore that probably you will thank me if I make this lecture a series of experimental demonstrations, rather than if I were to put endless mathematics on the board in order to explain what already is explained in books. (Cheers).

In order further to save your time and mine, I drew up a table of what I call aberrations, or what I so term, at any rate, whether the books do or not; and thanks to the kindness of the Editor and Pul lisher of The Optician I am able to give you this in printed forn. You will observe that I have drawn up five classes of aberrations. (See next page.) 


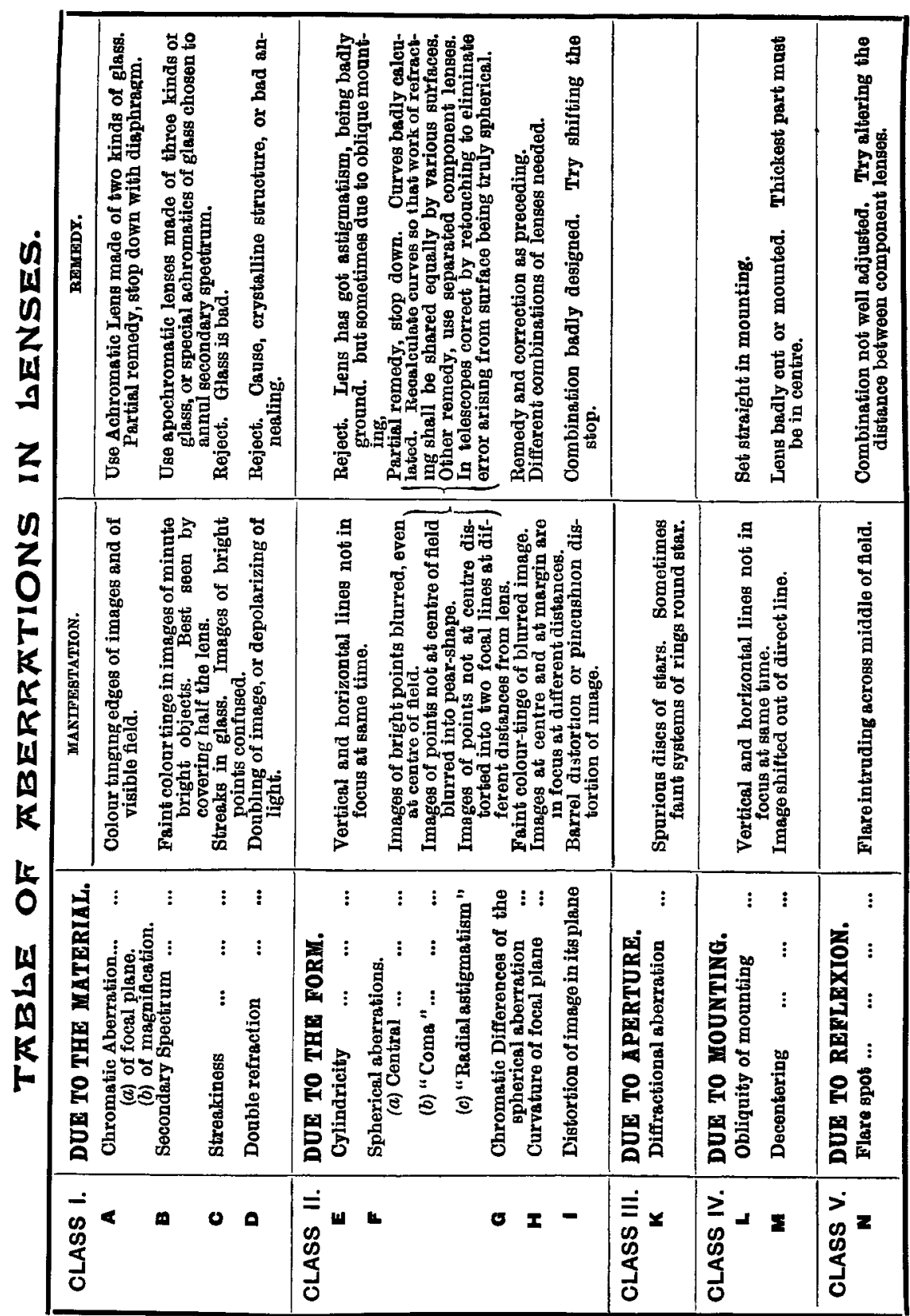

C 
Perhaps we might dispose of the last one first, and have nothing more to say about it. The only aberration that we need trouble about due to reflexion is that commonly called "flare," which manifests itself mostly in a spot occurring right in the middle of the field. One comes across it in photographic lenses. It is much the best seen by getting a photographic lens that is bad in this respect. I cannot show it to you any better, so will not say anything more about it; but I think I shall say something about all the others, and try to show you some of them.

Now, going back to Class I., the aberrations due to material. Every material of which a lens is made operates on the waves of light; and whether it be a converging lens or a diverging lens, all that it can possibly do is to alter the shape of the wave-fronts of the waves that come into it. If the waves are coming from a practically infinite distance, every front marching along parallel lines, when they go through the lens they no longer come out with flat fronts. If they come out with a bulging front, then they will diverge. Every wave marches in a direction at right angles to its own front. (Illustrating.)

And now, if for any reason, the wave comes out with a bulging front, it will go on marching at right angles to its own front in this way, and therefore spread as from a centre. This is the reason why we have a divergence, produced by a negative lens.

Let us inquire a little further as to why and how that bulging is formed, how is it the middle part gains on the edge part?-Glass is a retarding medium, light will not go through glass so fast as through air, and therefore if you interpose a piece of glass thin in the middle and thick at the edges, the middle part of the wave only having to go through the smaller thickness of glass, is not so much retarded as the outer portions, and the result is that the middle part gains upon them, and the wave comes out bulging and diverging; and on the contray, if your lens is thick in the middle and thin at the edges, the light going through the middle has to go through a greater thickness, consequently now the middle part is retarded more than the marginal parts, and the wave-front comes out concave.

Now that would give us a real focus as everybody knows; but if the glass-and of course this is true for all glasses-does not retard all different colours of light equally, then the focus will not be the same for all different colours; there will be a shorter focal length for that sort of light which is most retarded, and a longer for that kind of light which is the least retarded. And so we have what is 
called the aberration of colour, or chromatic aberration (No. A in the table) ; but, as a matter of fact, there are two different kinds which it is well to distinguish, which are very often not distinguished in books. A lens made of one piece of glass may produce a chromatic aberration in this sense, that it produces the image for different colours at different places. You have different foci when red or blue light is going through the lens. Here for instance (illustrating) it has its principal focus for red at a certain distance, and for yellow it has its principal focus at a little shorter distance from the lens; green light shorter, blue light still shorter and violet still shorter. You have different actual positions in space for the different foci of the different colours. That is what I have put down there under the sub-title (a). But you might have a lens with a different kind of chromatic aberration. You might have a lens such, that though all the focal planes were the same for the different colours, yet the images in that plane would be of different sizes. This arises from the circumstance that every thick lens has really more than one optical centre; you have, in fact, two so-called "equivalent points" which act as optical centres, and from which are measured the true focal lengths which determine the magnification. Now, it is conceivable that you might have an optical combination, bringing all different lights to a focus in the same focal plane, yet the widths of the images might be different. There always are, in fact, in a single lens two optical centres for red, as well as two for blue. Consequently you have a different magnification for a light of different colour. It is well to distinguish between these two sorts of chromatic aberration.

Everybody knows that you can correct in the main for chromatic aberration by combining together two lenses. You take a lens of crown and a correcting lens of flint; and that combination, if properly chosen, gives us an achromatic lens. But no achromatic lens can be achromatic for every colour of the spectrum; at the most it can only bring together two different colours-it may bring red and blue to focus exactly at the same place, but the yellow, green, orange and violet will not come exactly to the same place. This is because of what is called the irrationality of the dispersion. If you were to make a prism of any particular kind of glass and use that prism to produce a spectrum with the various lines of the spectrum spaced out as we know they are (illustrating), you would have a certain spacing out of those lines of the spectrum, the lines of different colours being differently spaced out. All kinds of glass crowd up the orange and 
the red too much in proportion to their wave-length. If you produce a spectrum by means of a diffraction grating of fine lines ruled on glass, the spectrum is quite different, the spacing of the lines being absolutely proportional to the wave-lengths. Glass prisms always crowd up the red and orange relatively to the green, blue and violet. In fact, in the spectrum produced by a glass prism, the yellow, which should be in the middle, is always squeezed along toward the red end. But all glasses do not produce the same kind of crowding. Some glasses will produce more crowdings in the red piece of the spectrum, other glasses in yellow and green, and consequently you do not get an exactly proportional distribution of the different colours ; and, therefore, because of the "irrationality" of the dispersion in different kinds of glass when used to bring together two particular kinds of light, there is always what we call a secondary spectrum. It is rather a stupid name: "residual spectrum" is better. You will see it in even the best achromatic lenses in the following way: Take your achromatic lens and use as the object a fine line of light; for instance, the line you might get by letting down the blinds and getting a slit against the sky. Another way is, of course, to make a slit in a piece of cardboard or a piece of paper and put a candle behind it. You get such a slit and a long way off you focus the image of that slit (illustrating). Now cover up half the lens with a piece of paper or anything else you like, taking care that the edge is vertical, if the bright line is vertical-if you are using a horizontal bright line, then you must cover up the bottom or the top half-and at once you will get a coloured tinge, red at one side and blue at the other. You can do it with any ordinary achromatic telescope objective. There are very good field glasses which illustrate this well. If you will focus a telescope on a bright line and cover up half the object glass, you will always be able to see this effect.

The eye is similarly shown to be not achromatic. Look at any bright light from a distance-say, a lamp-and then cover up half the pupil and you will at once perceive the colours of the spectrum, proving that the eye is not corrected for chromatic aberration.

Now, for the purpose of showing the achromatic aberration, I am proposing to use a very admirable lens which I bought second-hand in a marine store dealer's some years ago. I believe it was originally used for one of those small lighthouses on the quays at the port of Bristol-little lighthouses which they have, about $20 \mathrm{ft}$. high, to guide 
the ships going round the corners of the harbour. It is an admirable lens, because it has every defect under the sun. (Laughter.)

Now we will have some light from an electric arc lamp thrown upon this lens, and we will endeavour to see whether it is achromatic. If it is, we shall have a white, bright spot as the image. But the spot, though bright, is not the only thing; there is a flare round it, the coma, due to spherical aberration as we shall see presently. We put a sheet of paper at the focus, and find the spot tinged with red, with rather a bluish centre; or, putting it nearer or further off, we get a very undefined kind of thing. Now if we cover up the middle of the lens, so as to allow the edges to produce their defects, we find that the chromatic aberration is a great deal worse; that the red margin is a great deal redder; but still better if we cut off from the light that we are using all the middle part of the spectrum, all the yellow and the green, and use only the colours at the extremes, we shall have red at one end, possibly with some orange, and shall have at the other end only blue and violet. To do that we might use violet glass, or purple, or what I am going to use in this little tank, Condy's fluid, that is to say, a solution of permanganate of soda or potash with water. This is the method I usually adopt, as by this means these achromatic aberrations can be shown exceedingly well. Look at the bluish centre (illustrating).

We may possibly show that again in another way. If you will look at this diagram you will see that the red makes a kind of an outer cone round all the other colours. Supposing we block out the middle of the lens, there is a cone of darkness, then a cone of light; and according to where we put our screen we shall get a red centre with blue outside, on a blue centre with red outside. Now if behind the lens we make a cloud of dust particles in the space to reflect the light we shall probably be able to see this without using any screen at all (illustrating). I have a tank full of smoke here, which may also be used to show this effect; you see a distinct cone of red rays outside the others. Let us try with another lens.

I may remark in passing, about that residual spectrum due to the irrational dispersion of glasses, that with the introduction of so many new kinds of glass it has been found possible to partly, though not absolutely, compensate for the irrationality of dispersion; for, although all glasses are irrational, you may be able to pick out pairs of glasses which are about equally irrational. For instance, if you found a glass which crowded up the orange part of the spectrum more than other parts-a crown glass which crowded 
up the orange-you might also be able to find a flint glass which also crowded up the orange, and out of those two you could make a better achromatic lens than by taking that particular crown with a different flint. Indeed it is one of the chief advantages of the new glasses made in Jena-that you can correct more or less for all parts of a spectrum; you can get nearly all the colours right without any residual error. In Schott's catalogue you will find certain pairs recommended, because they are pairs that will work together in that way.

Here is a lens-a plano-convex single glass lens-and we will focus the beam nearly parallel on to it. If I cover up the greater part of the surface of the lens, so as only to show the marginal part, we shall exaggerate the aberration. Now if we cover it with purple glass we shall have this spot with a red margin round it (an orange-red margin with a purplish interior), or we shall have a purplish margin with an orange interior ; a red margin with a blue interior, or a blue margin with a red interior. (Cheers.)

Now we must go on to the next point-aberrations $\mathrm{C}$ and $\mathrm{D}-\mathrm{C}$, want of precision of focus, which one finds if the glass is streaky, in which case you can do nothing with the lens; and, D, the aberration which arises if your material, instead of being singly refracting, is double refracting. A double refracting material is commonly either a crystalline body, or, if it is glass, it is badly annealed. I have some lenses here which are made of crystalline bodies. There is a quartz lens, and there is another. This one I owe to the kindness of Mr. Dixey, who was on the look out for a "good bad one" for me. This quartz lens is beautifully wrong, the crystalline axis is right across the middle. The crystal is exactly what it ought not to be-cut parallel to the axis (the axis is marked on it) with the consequence that it actually produces double images. You do not see that very well, because it has rather deep curres, unless you neutralise it. Neutralise one of these doubly refracting lenses with an ordinary positive or negative lens, and examine; and you will find, if you look through the edge at any bright body, any bright line, you get two images. This one actually does produce two images by double refraction.

Double refraction may often occur to such a small extent that you do not see it at all. Here is a lens that I use quite often for projecting objects on the screen, and it is very good so long as one is not working with polarized light. But when one is projecting on the screen with polarized light, I cannot use it in the usual place somewhero near the object, and between the polarizer and the analyser. 
When there should be darkness there appears a faint whiteness right across the middle; in fact a white cross. One or other of the lenses in the combination is badly annealed: hence the combination depolarizes.

Now we come to the aberrations which are due to form-to the shape of the lens. We will suppose the material to be otherwise perfect, yet if the shape is imperfect for its purpose, then we shall not have accurate images formed. And the first error that I choose to name 'is the error of cylindricity-that is to say, the lens has been ground not spherical, as it ought to be, but with curvatures which are more or less cylindrical ; we shall, of course, have aberrations, and the aberration above all others which is present here, if we employ a lens that is cylindrical, is, of course, astigmatism. We shall not have the vertical and horizontal lines of an object come to the same focus in the same plane.

It is very easy to illustrate that, provided you take an object that shall be composed of vertical and horizontal lines. I will take this lens, as it happens to be the most convenient for my purpose, and I will use it to make an image of this object. The lines are simply scratches ruled upon a sheet of smoked glass. Here, on the little screen, we will make an image of that object with vertical and horizontal lines in focus at the same time. Now we will take the cylindrical lens and put it with this, and it will give to it the same property as if this itself had cylindricity of curvature. We find we can focus the horizontal lines. Somewhere else we shall be able to focus the vertical lines, but we cannot get them both in focus (illustrating); and therefore we do not get a sharp focus at any place at all. That is clearly an astigmatic lens. It is afflicted with astigmatism in the proper sense of the word, as the eye might be afflicted.

I will show that again in another way. Using a little screen to catch the images, of course with the spherical lens alone, we get a cone of light which comes down to a point, if we have it properly centred; you can see the cone on my scattering dust in the air as before. Now, if we put the cylindrical lens along with it, so as to make the combination astiomatic, what shall we have? A cone which flattens to a line in one particular place and then widens out again to a small circle, and then fiattens horizontally to a line at right angles to the former one. We have two focal lines and no focal point. That is what we may call pure astigmatism.

The effects of astigmatism of the eye are very well known, I have 
no doubt, to you all, and I have been trying for some time to find whether I could get pictures which would show to an audience how a man who had an astigmatic eye would see something-what was the particular effect of the aberration of the eye which possesses astigmatism. I found the very thing I wanted issued by Messrs. Taylor, Taylor and Hobson, as an illustration of the good and bad behaviour of lenses. This first picture is to illustrate simply a set of circles, black and white, as seen by an ordinary eye, an eye in good condition, and then the next one is a thing as seen by an astigmatic eye, with vertical parts in good focus and horizontal parts blurred.

The camera lens is never astigmatic in this sense. Right across the whole field you never have this aberration. In this sense, no telescope, no opera glass, no camera, is astigmatic. What they call an astigmatism in a camera is something quite different.

The next aberration due to form is this: If the form of the lens, the form to which it has been ground, is truly and perfectly spherical, then, because it is truly and perfectly spherical, there will be aberrations. I know that some people have got a curious idea-I think it is the people who do not think for themselves, or who do not read accurately-that spherical aberration is an effect which lenses show when they are not truly spherical! It is just the other way about-it is because they are truly spherical.

Suppose light is coming out from a point, and you want to focus it at some other point, and you are taking a lens which is intended to do the work of collecting the rays which come out, the lens in between must turn the convex wave-front into a concave one. Now you want all the rays to be brought down to a focus at the other point. Clearly those rays which are very near to the axis have only to be turned through a small angle. Those rays which are out further have to be turned round more, and those rays still further, still more; the lens has to turn round these central rays a very little; those a little further out still more and so on.

If you take a double convex lens, which will show the spherical aberration you want to produce, having its curves perfect portions of spheres, the marginal parts will turn the light round too much, and bring it to a focus too soon-sooner than the central parts. That is to say these portions are too curved; they ought to slope back more. This same difficulty occurs with reflecting telescopes-with the concave mirror of the telescope-and they get over it in that case by grinding away the edges so that they shall not form a perfect sphere 
and in refracting telescopes the object glasses are also corrected by retouching. They must make them too flat a curve to begin with, and gradually work them down, and not produce true sphericity; but this will not do for ordinary work, for small telescope lenses and opera glasses and spectacles; one cannot go fiddling with local retouching to alter the curves; you must make the best and the truest spherical surfaces you can, and therefore have got to "correct." Now what is the golden rule for spherical aberration? The golden rule for spherical aberration is very simple. It depends on what you want to correct. Suppose you are going to use a parallel beam, and you intend to bring it down to a focus. You should strive to arrange your lens so that the two surfaces, the surface where the light enters, and where it emerges, perform equal work. If you used a lens of this shape, for example (plano-convex) with the plane side towards the source, clearly the light would go in straight. The whole of the work of deflecting is given to the second surface. Turn the convex face round the other way; then you have some of the work of refracting done at the first surface and some at the second. That will give you a sharper image of the centre of the field for the case of parallel beams of light : but not perfect. For the most perfect simple lens in this sense, using crown glass, the ratio of the radii of curvature would be about 6 to 1 , with flint 5 to 1 , with diamond lenses 4 to 1 . This is the particular case of parallel light to be brought to a focus at one point. For the case I gave you first-bringing light from a point on one side to a point equally distant on the other-the work of refracting must also be divided equally between the two surfaces. The best lens for this purpose, the lens freest from spherical aberration, is the equi-convex.

Now this kind of correction for the middle of the field, which is commonly called spherical aberration, and is the correction so described in the books, is only one of the spherical aberrations due to the form of the surface, and it is put down here under the head of "(a) central." Correct this as well as you can; you do not absolutely remove the spherical aberration even by proportioning the radii as 6 to 1. The best course you can adopt is to divide the work equally between the two surfaces. Suppose you get for parallel light the correct curvatures of the lens. But even then, though it may be spherically as correct as possible, if you use that lens for anything other than the purpose it was designed for, then it is no longer spherically correct. You can correct spherically for but two points only of the axis. In fact you might correct it for any two conjugate points. 
Here is a drawing to illustrate how the different zones of a lens produce unequal effects, the marginal part turning the light round too much, making the focal length too short, and the middle parts making it too long. I wish to show you this. My big lens is admirable for the purpose of showing you how the rays are crossing one another (illustrating). I suppose it is pretty.obvious that we have a kind of hollow curve all round here, an inverted arch. That in itself is evidence of spherical aberration.

Look at these rays where they cross the diameter; crossing and crossing and crossing they form a kind of arch, a "caustic-curve," as the result of the greater steepness of those from the margin as compared with these from the centre. Now-we will cover up this lens so as to allow light from only certain parts to come through, and we will take first of all light from the centre and near the centre only; then, if I scatter some chalk dust into the air, you will see the middle ray. Smoke will help us here. We are using the middle of the lens, and the region near the middle, and you see where these rays cross, marking the principal focus of the lens. Now I will open another pair of holes which are wider out, and from this wider zone of the lens the focus is nearer in. If we have the rays all going at once, you will see how the caustic curve is built up.

Now so far I have spoken only of this spherical aberration as affecting the question of whether you can get a sharply defined image at the middle of the field. For telescope work that is all you want. For you can always so turn your telescope that the star you want tolook at comes to the middle of the field. But if you want to do anything more, if you want to look at sun spots on the disc of the sun you cannot have it all focused at once. As soon as you wish to measure the distance from star to star, at once you find that the correction which is suggested to give you no spherical aberration in the middle of the field leaves you with all sorts of aberrations everywhere else; in fact, when you have corrected the middle of the field for spherical aberration you have only got rid of the first term of the series of terms of which the aberrations consist.

The second term in the series of spherical aberrations is this,-—that if you examine all the parts other than the centre of the field you discover that points which ought to focus sharply in the outlying regions do not focus sharply. Suppose you get the lens so arranged that for a point-object right on the axis you get a sharp point-image. Leaving the centre of the field you try it with some object which does 
not lie on the axis, some object on one side, and try whether near in or far out you can get a sharp-image. You do not get it at any point. Instead of that you get things which are nearly oval in shape or like this or that (illustrated). These curious specks you get for the various. images of the oblique projections of points, if the lens is not corrected, or only corrected for the middle of the field. This goes under the general name of "coma." I suppose it is meant that it looks like a comma in shape, or a comet.

I propose to show you this defect of coma. I have arranged here a little constellation of stars, made of small incandescent lamps, so that we may have not only one point but a number of points to be put into the field of view at the same time. Now this plano-convex lens, which is not at all a bad one for a single lens, does not profess to have the best shape for correcting spherical aberration, it is not achromatic, it has all the defects under the sun, except, that is as far as I am aware, there is no cylindricity about it. Let it be used to give us the images. We will try and get it placed opposite the middle of the constellation so that we may have one star at any rate central in our field of view.

Now you notice that every one of those stars, even the middle one, is fringed by a round patch. That is the coma (illustrating). We have no sharp focus anywhere except of the stars about the middle, which are fairly sharp, but not absolutely so. You might have a lens perfectly corrected for the centre, yet all the oblique images have the coma. On turning the bulging side towards us we have the coma outward instead of inward, and the definition in the middle is worse than before.

Some forty years ago a certain learned astronomer, Seidel, gave a very elaborate theory of the five different kinds of spherical aberration. So far as I know Seidel's theory has never been published in England. It is to our industrious, patient colleagues in Germany that we have had to look for the development of the theory of spherical aberration. It is true that Airy and Coddington and Herschel made many important discoveries, but the theory of getting a true image in a flat field we owe to Seidel.

He enumerates the aberrations in the following way: there is the general error of incorrect focusing, of which the first term, when corrected, gives you sharp images at the centre. This is what is ordinarily called spherical aberration. Then there are remains of the other errors, of which the next one to get rid of is coma, and after 
that there remains an error which is called radial astigmatism which $\mathbf{I}$ will show you next. After you have got rid of radial astigmatism there remains an error called the curcature of the surface of the image. The image is formed on a curved surface, instead of on a flat surface, so that it cannot be in focus all over the screen or plate at once. If you had a curved film for photographing upon you could avoid this defect. You could have a lens so designed that it would give you an absolutely sharp image all over a curved plate. And that leaves the fifth and final aberration-the distortion of the image in its own plane, the pincushion shape or barrel shape, as the case may be. These five aberrations cannot be corrected except by following out what is indicated by mathematical calculations as to the terms of the five errors that I have named. The corrections are these: first, for spherical aberration corrected as to the centre; second, the correction for coma; third, for radial astigmatism; fourth, the correction of the curvature of the image; and fifth, for distortion of the image in its own plane.

Now to correct five successive errors of this kind and to correct at the same time for two chromatic aberrations, and to give a lens a definite fucal length means that you have got to fulfil eight conditions. You have five conditions imposed to get rid of the spherical aberrations; you have two conditions to fulfil to bring two colours together without achromatic aberration; and you have the eighth condition, namely, the prescribed focal length; that is to say, you cannot possibly produce a. perfect lens unless you have eight variables in the lens itself.

If you are going to nse two kinds of glasses, it gives you two variables. Then you may vary the different radii of the curvatures. If the lens is to be cemented, two of these radii are obliged to be the same, and you have, therefore, one variable the less. You cannot get rid of eight different conditions that are imposed upon you unless you have at least eight variable quantities. If you have three lenses, separate pieces of glass, you have there six surfaces, the curvature of any one of which you may alter; when you are designing the lens you have got two distances which you may alter, and you have the possibility of using three different kinds of glass. Or say two outside of the same kind, the middle one different, two kinds of glass, two distances, six surfaces. Ten variables, you see, and that is quite enough. Some designers attain the end by means of three or four lenses cemented together. Observe how it is attained in a photographic portrait lens. The whole system is composed of two membersfront lens and a back lens. Each of these is in turn composed of two 
or more single lenses made of different kinds of glass, usually cemented together, though sometimes the individual members of the back-lens are separated by a layer of air. Further, each member of the combination is separately corrected for chromatic aberration; and they are so designed, of such thicknesses and at such a distance apart that the spherical and distortional aberrations of one member compensate as far as may be the aberrations of the other member. Lastly, the central "stop" assists in equalizing the aberrations at different parts of the field, and improves the marginal definition, though at the expense of the general brightness of the image.

This study of aberrations is necessary for this simple reason. You need only correct for the middle of the field in telescopes. In field glasses you have a rather wider angle. For opera glasses you want a very wide field, but people are not so particular here so long as they get the middle defined all right. But when you come to wide-angle photography, where you must have a wide angle with a large aperture to get the greatest amount of light, and where, particularly for process work, you must have no distortion and no error of focusing, and, in fact, perfect correction right up to the margin of the wide field, then this study of the five aberrations is essential. It becomes an absolute necessity: and the good lenses which are designed by modern makers are practically designed deliberately by knowing what are these five spherical and two achromatic conditions which have to be fulfilled, as well as giving the prescribed focal length; and the calculations are based upon these five equations of Seidel.

Now we have got here a lens which produces coma and it shows you how it is produced (illustrating). These different rays strike against the different parts of the lens, and instead of being brought to meet there where they ought to meet seem to have met across over there in space. It is the caustic-curve of the long tails of the coma displayed out as you see. The width between the outside piece and the next zone of it, there, arises from the width between the rays from the outermost part and the next outermost part of the lens. It is this outer zone of the lens that is responsible for that streak (illustrating).

If you could, from this oblique pencil, cut out only these rays which are contributions to the central part, you would of course at once reduce the tail of the coma and get a much sharper image. The object of the stop is to prevent the oblique pencils going through the middle of the lens and to make them go through the other part of the lens. That is exactly the way they do, if you notice, in landscape lenses. 
The stop put in front lessens the coma. To cure the coma fully, you have to make sure that all zones of the lens have equal focal lengths. Then, following an earlier calculation of Petzval's, Seidel showed that to correct the radial astigmatism you have got to fulfil a very precise condition between the radii and the indices of refraction of the lenses that are used in combinations.

Suppose central aberration, coma, and radial astigmatism all to have been eliminated, then that leaves two of the five aberrations-- the curvature of the image and the distortion in the plane. 'The curvature of the image can be corrected in compound lenses by a very simple process, namely, by slightly separating the front and the back components from one another. The diagrams on the wall are intended to illustrate this.

The only remaining kind of defect is, then, distortion of the images. This can be got rid of by proper disposition so that there shall be no more magnification at the margin than there is at the centre.

I have here just a simple old-fashioned lantern microscope, and if I put a good lens on-not a very powerful one-we shall get, of course, a very fair image on the screen. Now, I change that good lens for one that I know to be a bad one, and at once you see we have a distortion. The images of straight lines are no longer straight.

With regard to spherical aberrations a great discovery was made a few years ago; and again, I have to say, made in Germany. They found that they could produce a crown glass, for instance, this one, with a remarkably high refractive index, 1.61. The distinction is not nowadays so much "low" and "high" index; it is whether the glass has great dispersion relatively to its refraction or not.

We have, nowadays, crown glasses that have actually a higher refractive index than some of the light flints, while at the same time having a lesser chromatic dispersion. This new.Jena glass is simply invaluable in permitting of achromatic combinations which give a far flatter field than was previously possible with the old kinds of glass.

There remains but one matter to be considered, viz., the aberration due to aperture, sometimes called the aberration of diffraction, a thing which is an immediate consequence of the wave nature of light. If you have a lens collecting the waves over a considerable area with a wide aperture, and the wave comes out, as I explained at the begining, concave, so as to meet at a point, you do not, even when every correction has been made, and everything is as perfect as possible from the optical point of view, have, and you never will have, an absolutely 
sharp point there as the image of a distant bright point. And for this simple reason, that the waves from the different parts of the wavefront will interfere with one another.

Diffraction effects on a certain scale depend upon what is the size of the waves you are working with, also upon the length of the focus, and upon the amount of the visible aperture. The wider the aperture, up to a certain point, the less you will have of this trouble; the smaller the aperture, again up to a certain point, the less you will have. Anything very small or very large is distinctly bad. You are never troubled with it, however, except when you are dealing with long telescopes, and very accurate definitions of small stars. Then you get small bright discs, with rings of light round them, instead of small bright points. I would have liked to show you this experimentally; but it $i_{s}$ an effect which cannot well be projected upon the screen. So I must content myself with pictures of the effect.

I have two or three photographed figures here showing the kinds of aberration which you get due to diffraction, due to the interference of the waves. You see a little round disc of a definite size, say about $1 / 100$ th of an inch, and then rings of coloured light round it, gradually getting greater and greater. It is an aberration which mars the finest work. You cannot get rid of the fact that light waves hare a definite length. Light waves interfere, and diffraction will be set up. Diffraction is not simply, as many people are in the habit of supposing, caused by light grazing against the edge of an aperture. Diffraction occurs wherever you have waves. When you have a large object you do not notice this at all. When you want to get accurate definition of these small objects, then this comes into play. The best account of it is in the article on "Optics" in the Encyclopædia Britannica, written by Lord Rayleigh, who is one of our greatest authorities on diffraction and diffraction effects.

I am very sorry that I have kept you so long on this. I am exceedingly sorry I have not been able to show you this last thing as well as I would like to have done, but I must ask you to kindly forgive me in that respect, and to be willing for once to take the will for the deed. (Loud cheers.) 